Fission prompt gamma-ray multiplicity distribution measurements and simulations at DANCE

A. Chyzh, C. Y. Wu, J. Ullmann, M. Jandel, T. Bredeweg, A. Couture, E. Norman

August 31, 2010 
This document was prepared as an account of work sponsored by an agency of the United States government. Neither the United States government nor Lawrence Livermore National Security, LLC, nor any of their employees makes any warranty, expressed or implied, or assumes any legal liability or responsibility for the accuracy, completeness, or usefulness of any information, apparatus, product, or process disclosed, or represents that its use would not infringe privately owned rights. Reference herein to any specific commercial product, process, or service by trade name, trademark, manufacturer, or otherwise does not necessarily constitute or imply its endorsement, recommendation, or favoring by the United States government or Lawrence Livermore National Security, LLC. The views and opinions of authors expressed herein do not necessarily state or reflect those of the United States government or Lawrence Livermore National Security, LLC, and shall not be used for advertising or product endorsement purposes.

This work performed under the auspices of the U.S. Department of Energy by Lawrence Livermore National Laboratory under Contract DE-AC52-07NA27344. 
LLNL-TR-452298

\title{
Fission prompt $\gamma$-ray multiplicity distribution measurements and simulations at DANCE
}

\author{
A. Chyzh and C.Y. Wu \\ Lawrence Livermore National Laboratory, Livermore, CA 94551 \\ T. Bredeweg, A. Couture, M. Jandel, and J. Ullmann \\ Los Alamos National Laboratory, Los Alamos, NM 87545 \\ E. Norman \\ Department of Nuclear Engineering, \\ U. of California at Berkeley, Berkeley, CA 94720
}

(Dated: September 2010)

\begin{abstract}
The nearly energy independence of the DANCE efficiency and multiplicity response to $\gamma$ rays makes it possible to measure the prompt $\gamma$-ray multiplicity distribution in fission. We demonstrate this unique capability of DANCE through the comparison of $\gamma$-ray energy and multiplicity distribution between the measurement and numerical simulation for three radioactive sources ${ }^{22} \mathrm{Na},{ }^{60} \mathrm{Co}$, and ${ }^{88} \mathrm{Y}$. The prospect for measuring the $\gamma$-ray multiplicity distribution for both spontaneous and neutron-induced fission is discussed.

Keywords: DANCE, BaF2, scintillator, GEANT4, fission gamma ray multiplicity.
\end{abstract}




\section{INTRODUCTION}

The measurement of the $\gamma$-ray energy and multiplicity distribution in fission is important for both basic and applied nuclear physics. These quantities are needed to validate the modeling of $\gamma$-ray production in the fission process and to advance the design for the reactor fuel cycle. The experimental status on this subject has been evaluated recently by Valentine [? ] and he pointed out that Brunson [? ] is the only one who formulates a mathematic model

for his measurement of the prompt $\gamma$-ray multiplicity distribution of the ${ }^{252} \mathrm{Cf}$ spontaneous fission using $8 \gamma$-ray detectors, plastic scintillators (127 mm diameter and $127 \mathrm{~mm}$ length) together with a fission fragment detector. With the advantage of a modern $4 \pi$ high-efficiency and high-granularity $\gamma$-ray detector array one has an opportunity to renew this effort to measure these important quantities. However, the direct comparison of the fission $\gamma$-ray energy and multiplicity distribution between measurement and prediction is difficult since it is complicate to restore the true distributions from experimental data, and the reasons are:

1. the strong energy dependence of the detector response for the $\gamma$-ray efficiency and multiplicity;

2. many nuclear species are involved in the fission process.

Alternatively, one can compare these quantities between measurement and prediction after applying corrections for detector response. Unfortunately, the predicted $\gamma$-ray energy and multiplicity for fission is not readily available at present time. Therefore, even this alternative is not practical for studying these quantities. However, the $\gamma$-ray multiplicity distribution can be measured with sufficient accuracy if the detector response for the $\gamma$-ray efficiency and multiplicity is insensitive to the energy. Here we present one such detector system for measuring the fission prompt $\gamma$-ray multiplicity distribution.

\section{DANCE ARRAY}

DANCE [? ] (Detector for Advanced Neutron Capture Experiments) is a $\gamma$-ray calorimeter and designed to study the neutron-capture reactions on small quantities of radioactive and/or rare stable nuclei. These reactions are important for the radiochemistry applications and studying element production in stars. In the following sections we are going to present 
unique features of this detector array through the comparison between several measurements and simulations of the 3 radioactive sources, ${ }^{22} \mathrm{Na},{ }^{60} \mathrm{Co}$, and ${ }^{88} \mathrm{Y}$.

\section{GEANT4 model of DANCE}

The DANCE response to $\gamma$ rays was studied using the Monte Carlo simulation code GEANT4. The GEANT4 model of DANCE was written by Marian Jandel [? ] in 2007 and was tested extensively at LANL, LLNL, TUNL, and NCSU. The details of DANCE that are captured in the GEANT4 model include:

- 160 crystals of $\mathrm{BaF}_{2}$. Each crystal is wrapped with the PVC foil $0.7 \mathrm{~mm}$ thick. 5 shapes of crystals are of the same solid angle coverage from the target position inside DANCE.

- 160 photo multiplier tubes (PMT). Each PMT is a hollow cylinder made of aluminum.

- The aluminum beam pipe that goes through the middle of DANCE replacing 2 of $\mathrm{BaF}_{2}$ crystals.

- Enriched ${ }^{6} \mathrm{LiH}$ moderator with density $0.85 \frac{\mathrm{g}}{\mathrm{cm}^{3}}$. It is placed in the middle of DANCE and, thus, surrounds the target.

- The target is assumed to be a point source that emits $\gamma$-ray isotropically.

The input file is an ASCII formatted file that contains the $\gamma$ cascades: multiplicity and individual photon energies. The energy deposited in the crystals for each $\gamma$ ray in a given cascade is simulated and statistics are collected into spectra of individual $\gamma$-ray energy $\mathrm{E}_{\gamma}$ and total cascade energy $\mathrm{E}_{\Sigma}$ :

$$
E_{\Sigma}=\sum_{i=1}^{M} E_{\gamma i}
$$

where $M$ is the $\gamma$-ray multiplicity.

The simulated energy threshold, $150 \mathrm{keV}$, is the same one used in the experiments when measuring calibration sources ${ }^{22} \mathrm{Na},{ }^{60} \mathrm{Co}$, and ${ }^{88} \mathrm{Y}$. A Gaussian distribution with FWHM $=$ $100 \mathrm{keV}$ for the threshold is added on an event by event basis to simulate fluctuations of the threshold.

For more details on how the GEANT4 model of DANCE works see Ref. [? ]. 


\section{CALIBRATION SOURCES}

To validate this model, we simulate 3 calibration sources: ${ }^{22} \mathrm{Na},{ }^{60} \mathrm{Co}$, and ${ }^{88} \mathrm{Y}$. The 2006 and 2007 experimental data sets from these sources are used for comparison with the simulations. The calibration sources are better than beam experiments because they emit no neutron background, thus, the experimental data is much cleaner and contains only environmental background and internal background of the ${ }^{226} \mathrm{Ra}$ decay chain inside the $\mathrm{BaF}_{2}$ crystal. ${ }^{226} \mathrm{Ra}$ impurity is present in Ba because these two are chemical homologue. These backgrounds are mostly made of the $\mathrm{M} \gamma=1$ events. The calibration sources deliver the $\mathrm{M} \gamma=1, \mathrm{M} \gamma=2$, and $\mathrm{M} \gamma=3$ events to DANCE, which are almost free of any background and, thus, can be used to validate the model for the $\mathrm{E}_{\gamma}$ and $\mathrm{E}_{\Sigma}$ energy response.

$10^{6}$ decays are simulated for each calibration source. The $\gamma-\gamma$ angular correlations are neglected. The $\beta^{+}$decay of ${ }^{22} \mathrm{Na}$ decay is replaced with 2 photons at $511 \mathrm{keV}$, in reality the $511 \mathrm{keV}$ photons emit be back-to-back, but in the simulation we approximate them as isotropically emitted photons.

\section{${ }^{60}$ Co source}

Figure 1 shows the decay scheme of ${ }^{60}$ Co taken from [? ]. The most abundant channel is the one with ${ }^{60} \mathrm{Ni}$ deexciting from the $2505.765 \mathrm{keV}$ level to the $1332 \mathrm{keV}$ level and then to the ground state via 2 -rays of 1173 and $1332 \mathrm{keV}$. Each of the simulated events for ${ }^{60} \mathrm{Co}$ is a $\mathrm{M}=2$ event with 1173 and $1332 \mathrm{keV}$, thus, the $\gamma$-multiplicity is always 2 for this source, no other decay channels were simulated.

The figures 2, 5, 3, and 6, show simulated $\mathrm{E}_{\sum}$ spectra and experimental ones for different values of cluster multiplicity $\mathrm{M}_{c l}$. All the experimental spectra are normalized by a single number $\mathrm{K}_{\text {norm }}\left({ }^{60} \mathrm{Co}\right)$, thus, the ratio between spectra of different $\mathrm{M}_{c l}$ remains unchanged. The normalization coefficient $\mathrm{K}_{\text {norm }}\left({ }^{60} \mathrm{Co}\right)$ is calculated by normalizing the experimental and simulated $\mathrm{M}=2$ spectra in the vicinity of the $\mathrm{E}_{\sum}=1.17+1.33=2.5 \mathrm{MeV}$ peak. Then $\mathrm{K}_{\text {norm }}\left({ }^{60} \mathrm{Co}\right)$ is applied to the other ${ }^{60} \mathrm{Co}$ experimental spectra.

The figure 4 shows the $\mathrm{E}_{\sum}$ spectra summed for $\mathrm{M}=1$ and higher. The figure 7 is the sum of $\mathrm{E}_{\sum}$ spectra for $\mathrm{M}=2$ and higher.

The agreement between experiment and simulation at $\mathrm{M} \gamma=1$ is rather poor because 


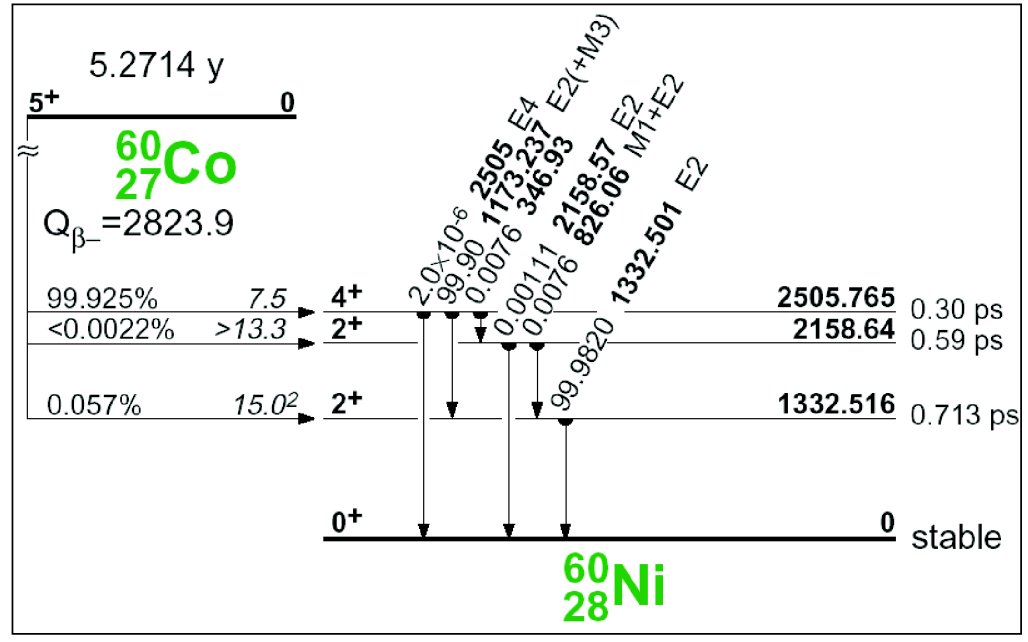

FIG. 1: The decay scheme of ${ }^{60} \mathrm{Co}$.

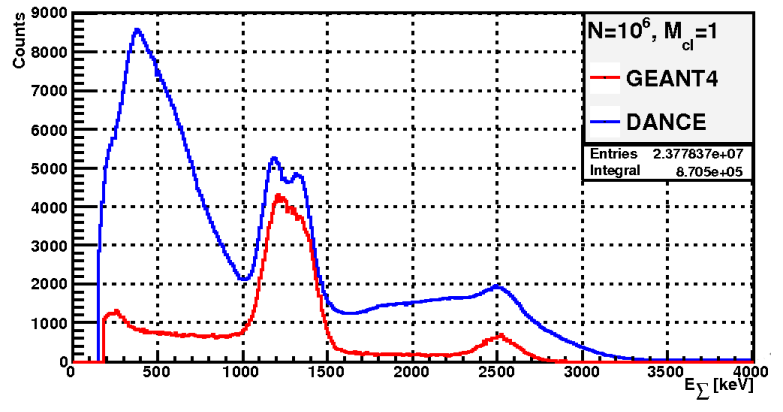

FIG. 2: $\mathrm{E}_{\sum}$ spectra of ${ }^{60} \mathrm{Co}$ source: $\mathrm{M}=1$.

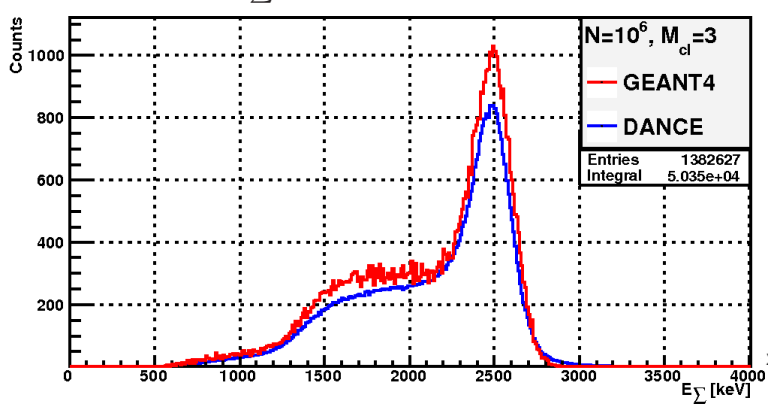

FIG. 3: $\mathrm{E}_{\sum}$ spectra of ${ }^{60} \mathrm{Co}$ source: $\mathrm{M}=3$.

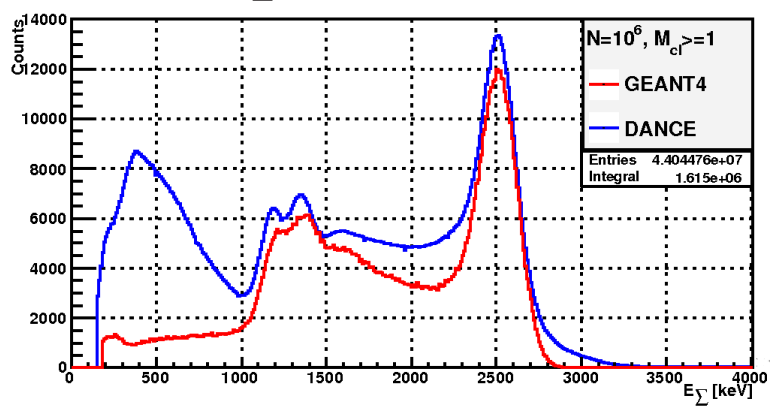

FIG. 4: $\mathrm{E}_{\sum}$ spectra of ${ }^{60}$ Co source: $\mathrm{M} \geq 1$.

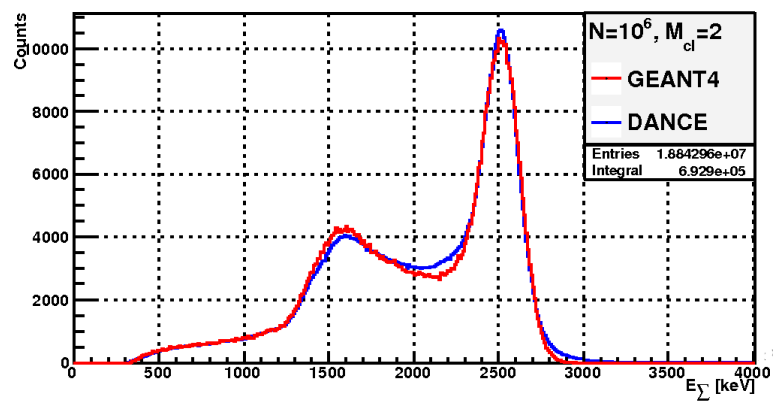

FIG. 5: $\mathrm{E}_{\sum}$ spectra of ${ }^{60}$ Co source: $\mathrm{M}=2$.

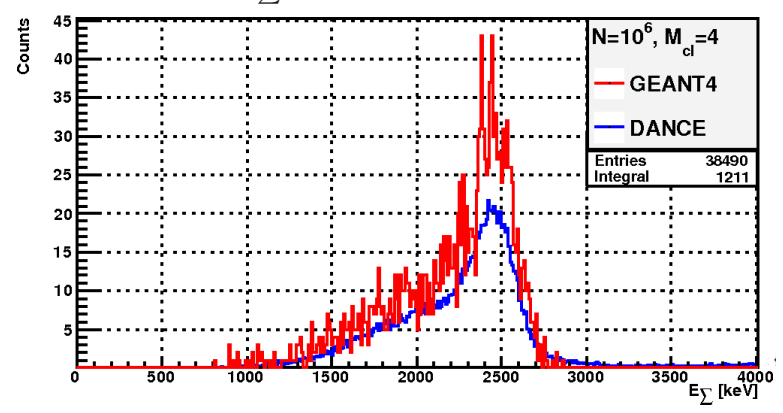

FIG. 6: $\mathrm{E}_{\sum}$ spectra of ${ }^{60} \mathrm{Co}$ source: $\mathrm{M}=4$.

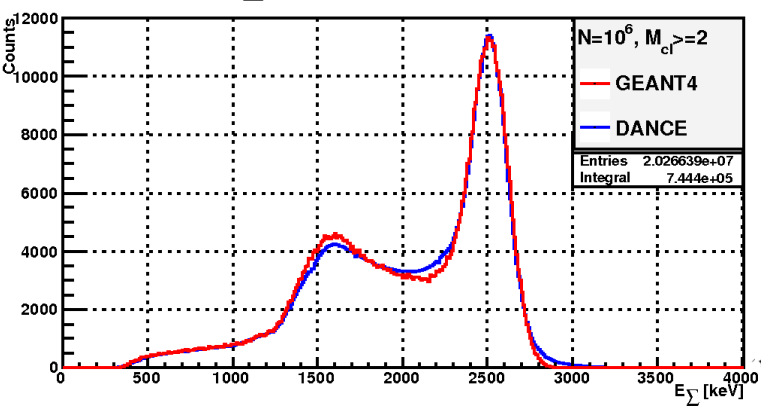

FIG. 7: $\mathrm{E}_{\sum}$ spectra of ${ }^{60}$ Co source: $\mathrm{M} \geq 2$. 
of high level of environment background inevitably presenting when measuring ${ }^{60} \mathrm{Co}$ and low energy background inside $\mathrm{BaF}_{2}$ crystals from the ${ }^{226} \mathrm{Ra}$ decay chain, it has several $\beta$ emitters. Although the beam was off during the measurements, the backgrounds are made of mainly $\mathrm{M} \gamma=1$ events. The internal $\alpha$ background is well separated by applying pulse shape discrimination (PSD) technique. The $\mathrm{M}=2$ spectra agree well because they were directly normalized. The $\mathrm{M}=3$ and $\mathrm{M}=4$ spectra agree within $5 \%$ and $10 \%$ of their integrals The $\mathrm{M} \geq 1$ spectra agree well only around the $2.5 \mathrm{MeV}$ peak, in the lower energy region the presence of background cannot be easily simulated. From the ${ }^{60} \mathrm{Co}$ comparison one can conclude that the GEANT4 model of DANCE simulates the $\mathrm{M}=2$ events with $\mathrm{E}_{\gamma}=1.17$ and $1.33 \mathrm{MeV}$ very well.

\section{${ }^{22} \mathrm{Na}$ source}

The decay scheme of ${ }^{22} \mathrm{Na}$ is shown at Fig. 8. The probability for the $\beta^{+}$decay of this isotope is $90.5 \%$ and the remaining $9.5 \%$ is for the electron capture decay. The input file of $\gamma$-cascades for ${ }^{22} \mathrm{Na}$ simulation contains:

- $90.5 \%$ of $\mathrm{M}=3$ events with $\gamma$-energies 511,511 , and $1274.5 \mathrm{keV}$.

- $9.5 \%$ of $\mathrm{M}=1$ events with $1274.5 \mathrm{keV}$ energy.

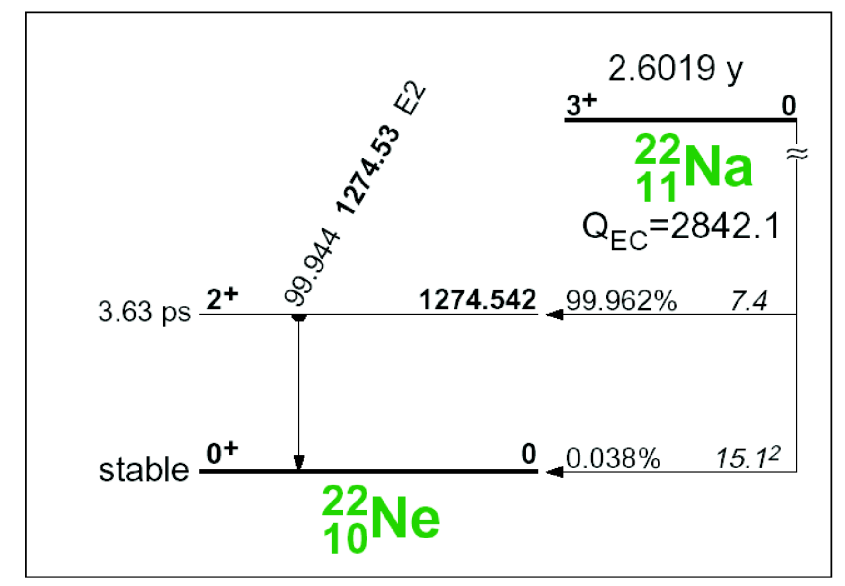

FIG. 8: The decay scheme of ${ }^{22} \mathrm{Na}$.

The figures 9, 12, 10, and 13, show comparison of simulated and experimental ${ }^{22} \mathrm{Na}$ data for different values of $\mathrm{M}_{c l}$. The normalization $\mathrm{K}_{\text {norm }}\left({ }^{22} \mathrm{Na}\right)$ between simulation and 
experiment is calculated for the $511+511+1274 \mathrm{keV}$ sum peak for the $\mathrm{M}=3$ spectra and applied to the ${ }^{22} \mathrm{Na}$ experimental spectra of other multiplicities. The background at $\mathrm{M}=1$ is quite similar to that of ${ }^{60} \mathrm{Co}$.

The differences in the $M=3$ and $M=4$ spectra integrals are $\sim 10 \%$. One reason for that is because the $\beta^{+}$-particle is not simulated accurately. In reality the positron with high probability leaves the target location and hits the Al beam pipe away from the target location, which leads to the emission of two $511 \mathrm{keV}$ photons in opposite directions and from this location the efficiency is not the same for all the $\mathrm{BaF}_{2}$ crystals. In simulation the positron is replaced with two $511 \mathrm{keV}$ photons that are isotropically emitted from the target location, so the efficiency is the same for all the crystals. Another reason for $\sim 10 \%$ disagreement is the residual presence of background in the $\mathrm{M} \gamma=2$ experimental spectrum. As was mentioned above the ${ }^{226}$ Ra decay chain produces $\alpha$-particles that can be removed by PSD and $\beta$-particles that are hard to remove, the $\alpha$ and $\beta$ are mainly $\mathrm{M}=1$ events, but small fraction of them is registered as $M=2$ events.

\section{${ }^{88} \mathbf{Y}$ source}

Figure 15 represents the decay channels of ${ }^{88} \mathrm{Y}$ :

- $99.8 \%$ - electron capture;

- $0.2 \%-\beta^{+}$-decay;

The simulation neglects the $\beta^{+}$-decay and considers only electron capture that leads to ${ }^{88} \mathrm{Sr}$. Two major channels of the ${ }^{88} \mathrm{Y} \rightarrow{ }^{88} \mathrm{Sr}$ decay are simulated as follows:

- $94.5 \%$ of $\mathrm{M}=2$ events -2 photons of the 898 and $1836 \mathrm{keV}$ energies;

- $5.5 \%$ of $\mathrm{M}=1$ events -1 photon of $1836 \mathrm{keV}$;

The figures $16,19,17$, and 20, show the comparison of simulated and experimental $\mathrm{E}_{\Sigma}$ spectra. The normalization was calculated for the $M=2$ spectra, since $M=2$ is the most frequent type of events, and then was applied to other multiplicities. The agreement between simulation and experiment at $\mathrm{M}=2$ and $\mathrm{M}=3$ is within $\sim 5 \%$ and at $\mathrm{M}=4$ it is $\sim 10 \%$. The biggest disagreement is at $\mathrm{M}=1$ because of ambient background during the ${ }^{88} \mathrm{Y}$ measurement. 


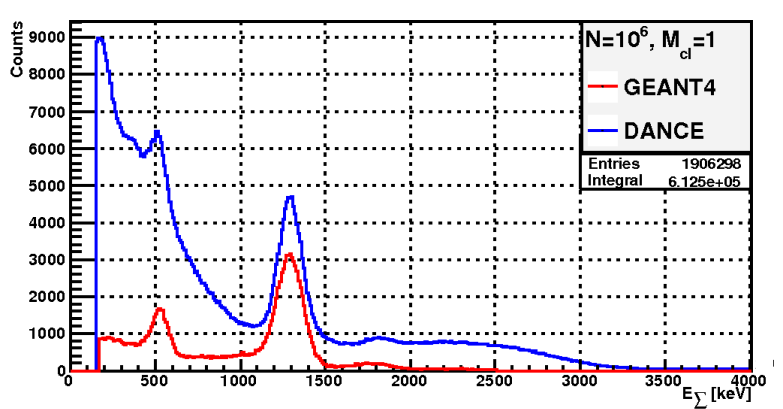

FIG. 9: $\mathrm{E}_{\sum}$ spectra of ${ }^{22} \mathrm{Na}$ source: $\mathrm{M}=1$.

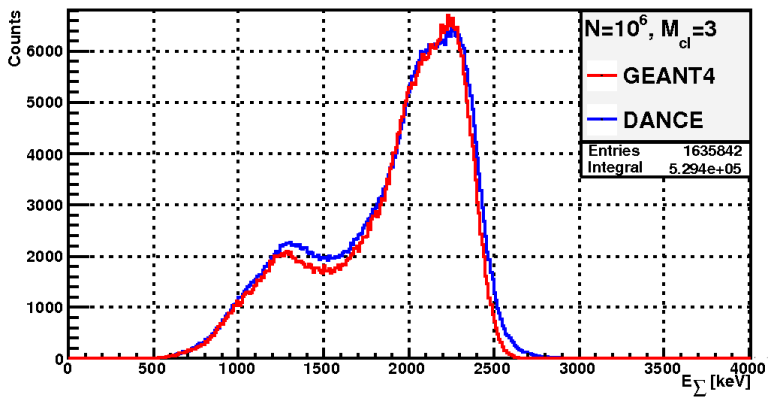

FIG. 10: $\mathrm{E}_{\sum}$ spectra of ${ }^{22} \mathrm{Na}$ source: $\mathrm{M}=3$.

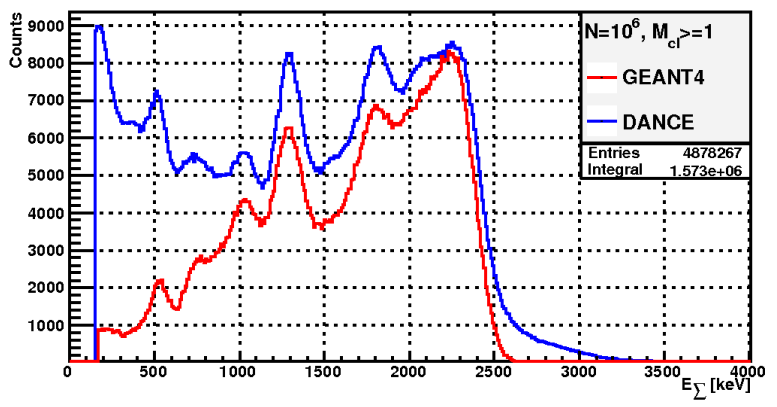

FIG. 11: $\mathrm{E}_{\sum}$ spectra of ${ }^{22} \mathrm{Na}$ source: $\mathrm{M} \geq 1$.

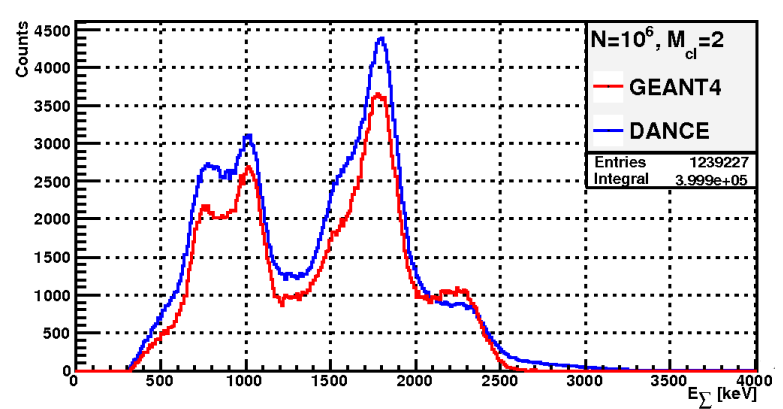

FIG. 12: $\mathrm{E}_{\sum}$ spectra of ${ }^{22} \mathrm{Na}$ source: $\mathrm{M}=2$.

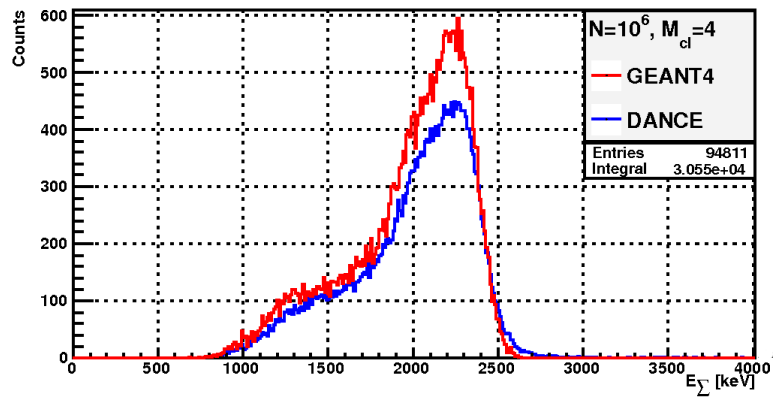

FIG. 13: $\mathrm{E}_{\sum}$ spectra of ${ }^{22} \mathrm{Na}$ source: $\mathrm{M}=4$.

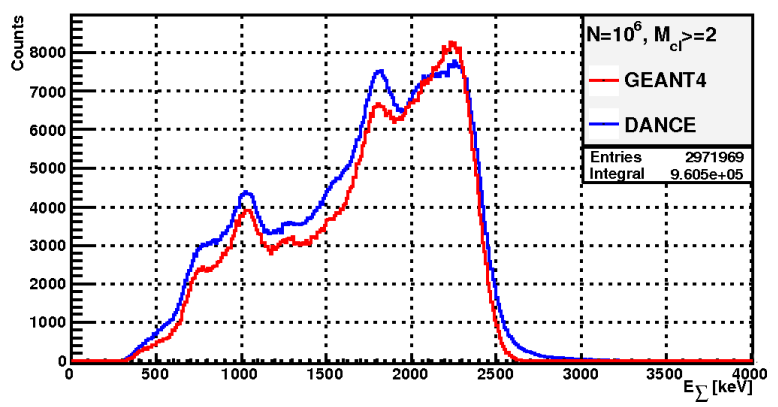

FIG. 14: $\mathrm{E}_{\sum}$ spectra of ${ }^{22} \mathrm{Na}$ source: $\mathrm{M} \geq 2$.

This disagreement is worse than those for ${ }^{60} \mathrm{Co}$ and ${ }^{22} \mathrm{Na}$ because the ${ }^{88} \mathrm{Y}$ source was weaker at the time of measurement.

The figures 18 and 21 are spectra comparisons for $\mathrm{M} \geq 1$ and $\mathrm{M} \geq 2$ respectively. The agreement at $\mathrm{M} \geq 2$ is within $\sim 5 \%$ which is almost the same as for $\mathrm{M}=2$ because most of the statistics comes from the $\mathrm{M}=2$ events. The "too good to be true" agreement of the peaks at the $898+1836=2734 \mathrm{keV}$ energy is the evidence that the DANCE response to the $\gamma$-ray can be simulated confidently with this GEANT4 model. 


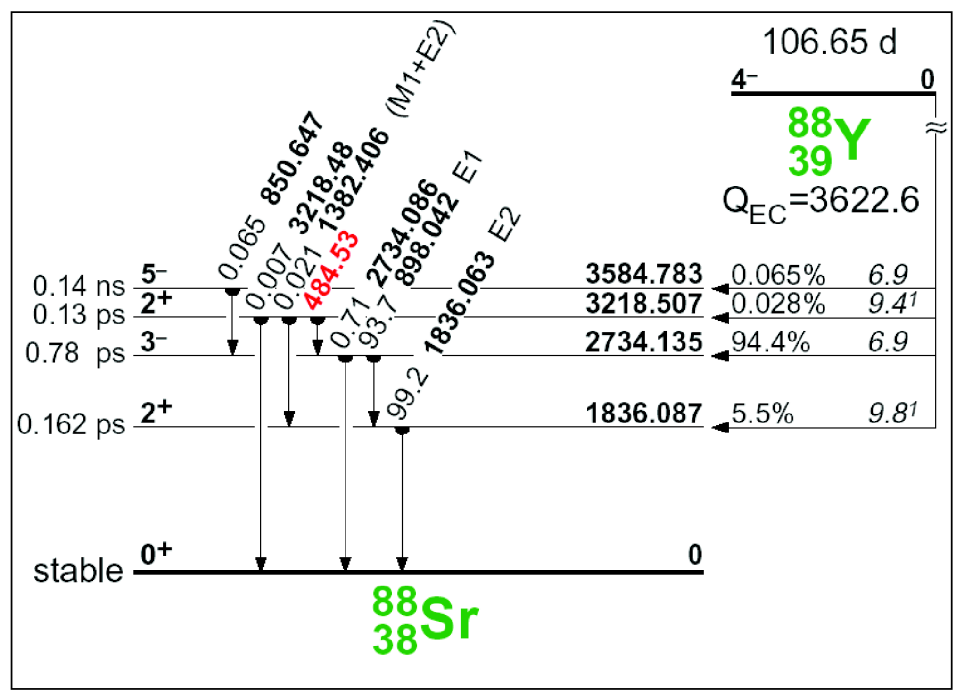

FIG. 15: The decay scheme of ${ }^{88} \mathrm{Y}$.

\section{MULTIPLICITY AND ENERGY RESPONSE}

The simulations of the calibration sources ${ }^{60} \mathrm{Co},{ }^{22} \mathrm{Na}$, and ${ }^{88} \mathrm{Y}$, serve as a validation for the GEANT4 model of DANCE. In this section we describe how the DANCE energy and multiplicity responses to $\gamma$ rays were simulated and what results we obtained.

\section{$\gamma$-ray energy response}

The figure 22 shows the simulated responses of DANCE to different $\gamma$-ray energy $\mathrm{E}_{\gamma}$. The $\mathrm{E}_{\sum}$ spectrum is a total energy deposited into $\mathrm{BaF}_{2}$ crystal. Each $\mathrm{E}_{\sum}$ spectrum corresponds to a single primary $\gamma$-ray with energy $\mathrm{E}_{\gamma}$. The range of $\mathrm{E}_{\gamma}$ spans from 0.3 to $10 \mathrm{MeV}$, each $\mathrm{E}_{\gamma}$ was simulated with $10^{5}$ events of $\mathrm{M}_{\gamma}=1$, isotropically distributed from the center of DANCE, the $150 \mathrm{keV}$ threshold was applied to the pulse height of each $\mathrm{BaF}_{2}$ crystal. The analysis of these spectra revealed some properties of DANCE:

- the total efficiency $\epsilon_{\text {tot }}$ for detecting a single $\gamma$-ray for a given energy $\mathrm{E}_{\gamma}$ lies within 84.5 - $88.0 \%$, i.e., the DANCE response is almost independent of the photon energy, this is the most important property of the DANCE response;

- the ratio $\frac{\text { photopeak }}{\text { total }}$ is also nearly independent of the energy, it lies within $53-56 \%$, which means that the shape of the $\mathrm{E}_{\sum}$ spectrum at $\mathrm{M}_{\gamma}=1$ is very much independent 


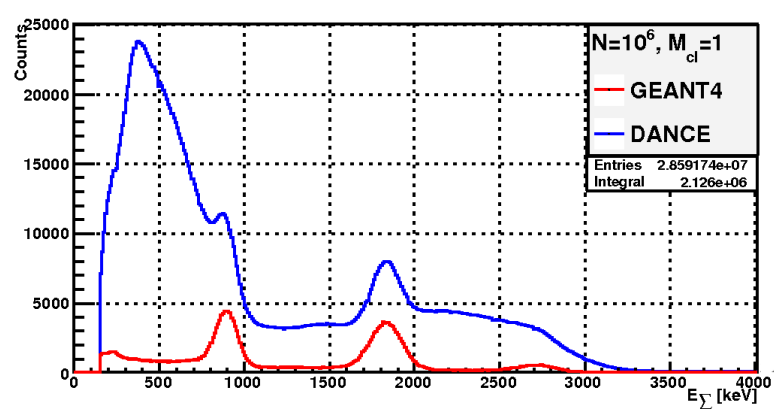

FIG. 16: $\mathrm{E}_{\sum}$ spectra of ${ }^{88} \mathrm{Y}$ source: $\mathrm{M}=1$.

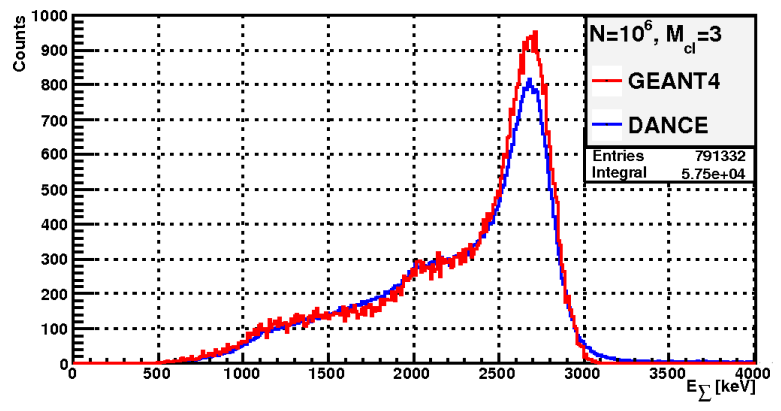

FIG. 17: $\mathrm{E}_{\sum}$ spectra of ${ }^{88} \mathrm{Y}$ source: $\mathrm{M}=3$.

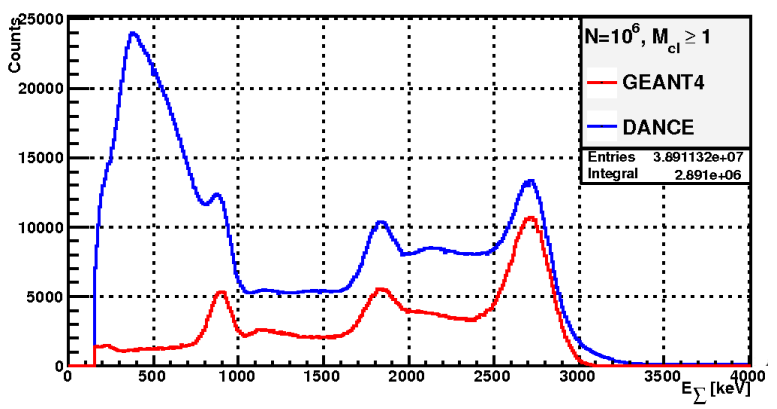

FIG. 18: $\mathrm{E}_{\sum}$ spectra of ${ }^{88} \mathrm{Y}$ source: $\mathrm{M} \geq 1$.

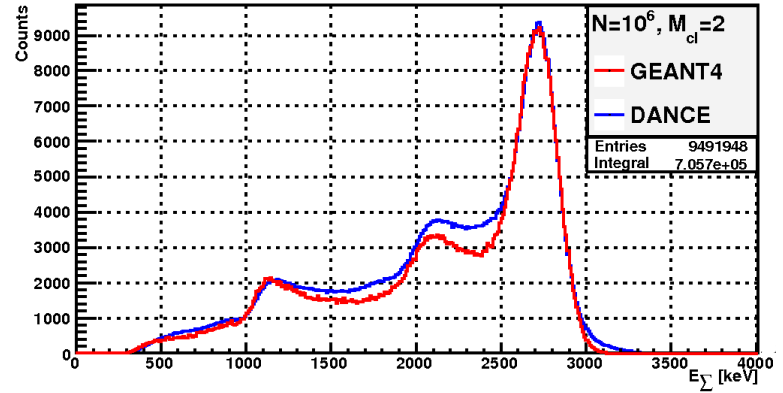

FIG. 19: $\mathrm{E}_{\sum}$ spectra of ${ }^{88} \mathrm{Y}$ source: $\mathrm{M}=2$.

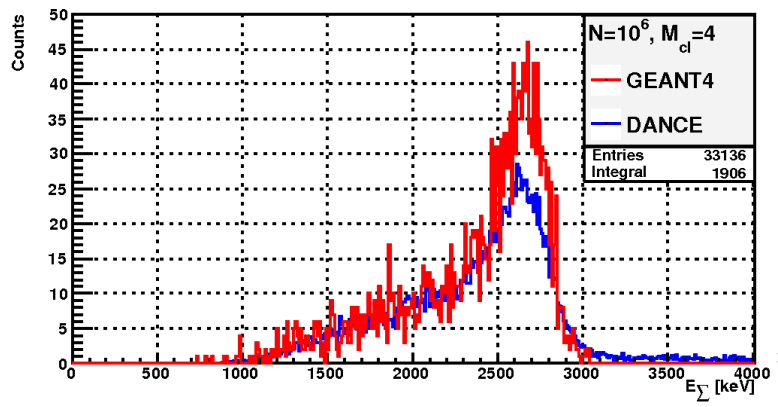

FIG. 20: $\mathrm{E}_{\sum}$ spectra of ${ }^{88} \mathrm{Y}$ source: $\mathrm{M}=4$.

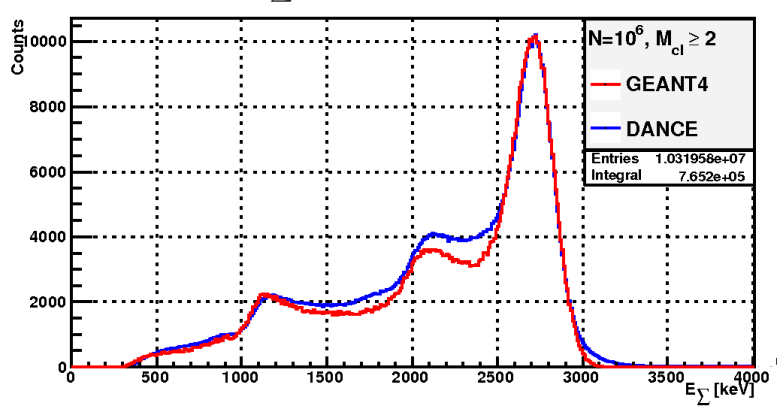

FIG. 21: $\mathrm{E}_{\sum}$ spectra of ${ }^{88} \mathrm{Y}$ source: $\mathrm{M} \geq 2$.

of $\mathrm{E}_{\gamma}$.

The efficiency reaches minimum value of $\epsilon_{\text {tot }}=84.5 \%$ at $\mathrm{E}_{\gamma} \simeq 5 \mathrm{MeV}$ and maximum $\epsilon_{\text {tot }}=88.5 \%$ at $\mathrm{E}_{\gamma} \simeq 1 \mathrm{MeV}$. The efficiency drops off "sharply" at low $\mathrm{E}_{\gamma}$ due to the 150 $\mathrm{keV}$ threshold, but still the difference between $\epsilon_{\text {tot }}(0.5 \mathrm{MeV})$ and $\epsilon_{\text {tot }}(2 \mathrm{MeV})$ is only $1 \%$.

This is an important property of the DANCE response to $\gamma$-rays. Most of the $\gamma$-rays from neutron capture have energies $\mathrm{E}_{\gamma} \leq 2-3 \mathrm{MeV}$, it is only the $(n, f)$ and $(x, f)$ reactions where we can see $\mathrm{E}_{\gamma}$ up to $8 \mathrm{MeV}$. 

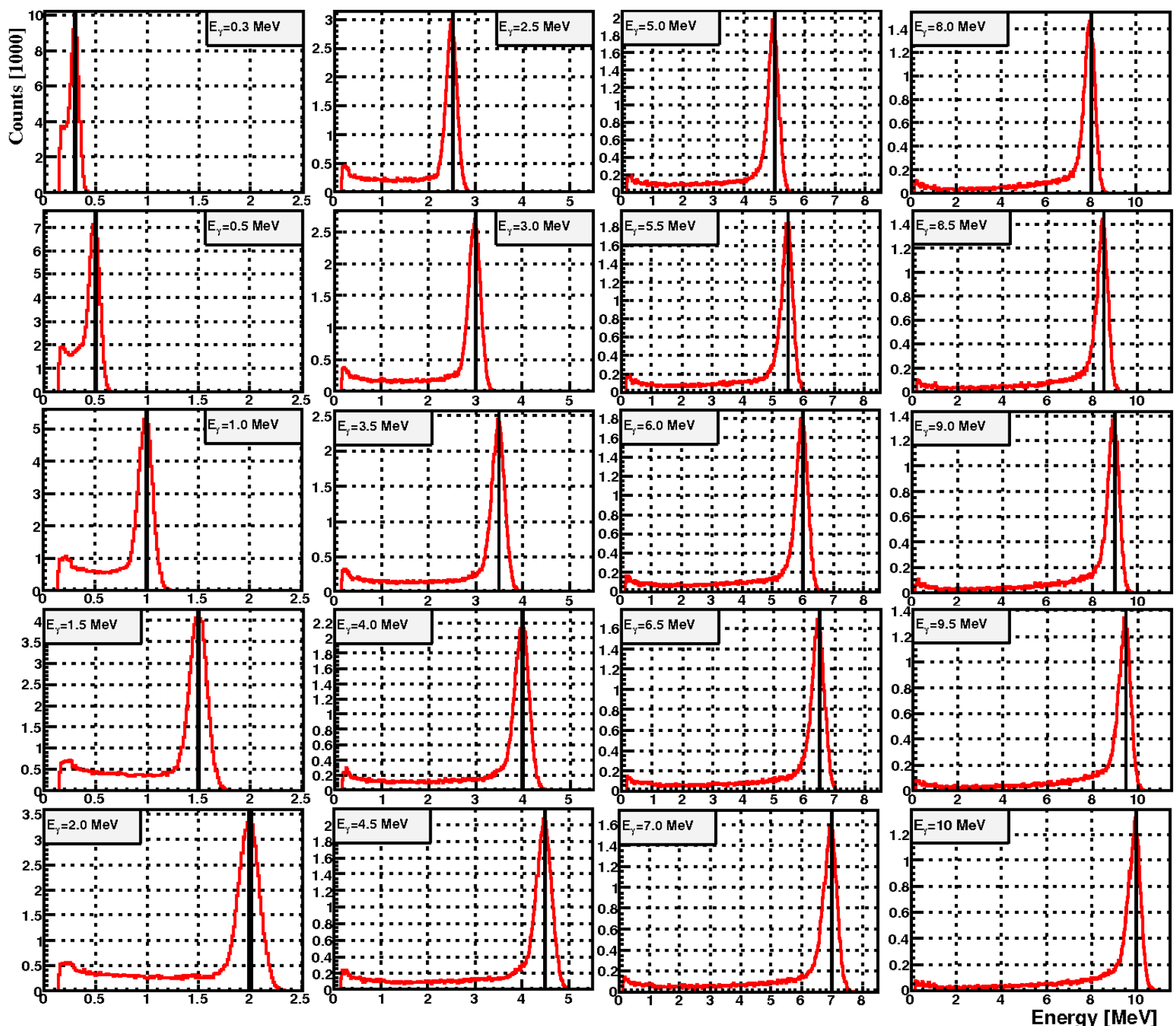

FIG. 22: Total energy deposited $\mathrm{E}_{\sum}$ for primary $\gamma$-ray energies from 0.3 to $10 \mathrm{MeV}$. Black line indicates primary $\gamma$-energy $\mathrm{E}_{\gamma}, 10^{5}$ events at $\mathrm{M}_{\gamma}=1$. Red line is the total energy, $\mathrm{E}_{\sum}$, deposited into DANCE.

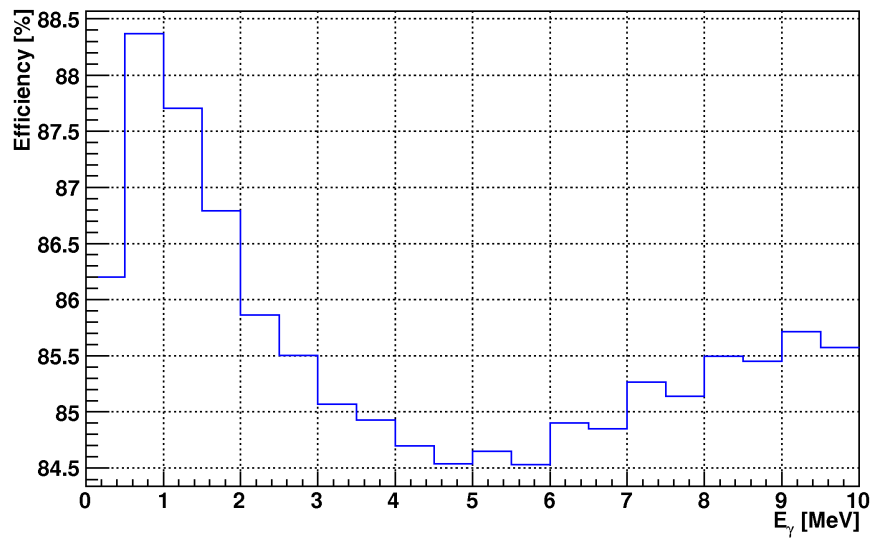

FIG. 23: Efficiency of detecting single $\gamma$-ray with a given energy 
$\gamma$-ray multiplicity response

There are two methods to deduce $\gamma$-ray multiplicity $\mathrm{M}_{\gamma}$ using DANCE:

- crystal multiplicity $\mathrm{M}_{c r}$ - number of crystals firing within time coincidence window $\left(\mathrm{T}_{0} ; \mathrm{T}_{0}+\Delta \mathrm{T}\right)$ above threshold; for calibration sources the time window was $\Delta \mathrm{T}=3$ ns;

- cluster multiplicity $\mathrm{M}_{c l}$ - number of groups of crystals, where each such group consists of adjacent crystals firing within the same time coincidence window above threshold.

From our previous experience [? ] with DANCE we know that the cluster multiplicity $\mathrm{M}_{c l}$ resembles the folded $\gamma$-ray multiplicity $\mathrm{M}_{\gamma}$ much better than the crystal multiplicity $\mathrm{M}_{c r}$ does up to $\mathrm{M}=20$ at least, because $\mathrm{M}_{c l}$ is cleaner of background than $\mathrm{M}_{c r}$, but eventually clusters begin to overlap and, thus, $\mathrm{M}_{c r}$ might be a better choice at $\mathrm{M}=30$ and higher.

The figure 24 shows the simulated $\mathrm{M}_{c l}$ response of DANCE to $\mathrm{M}_{\gamma}$. Each plot contains 3 spectra, each spectrum was simulated with $10^{5}$ mono energetic $\gamma$-rays: $\mathbf{0 . 5 1 1} \mathbf{M e V}$ in black, $1 \mathrm{MeV}$ in blue, $2 \mathrm{MeV}$ in red. Let's take a look at the $\mathrm{M}_{\gamma}=1$ plot. The true $\gamma$-ray multiplicity $\mathrm{M}_{\gamma}=1$, the total efficiency $\epsilon_{\text {tot }}(0.511 \mathrm{MeV})=88.3 \%$, from which $\epsilon(M=1)=85 \%$, $\epsilon(M=2)=2 \%$, and $\epsilon_{M=0}=13 \%$. Thus, the measured median $<\mathrm{M}_{c l}>=0.92$ corresponds to the real $\mathrm{M}_{\gamma}=1$.

The fact that one can misidentify the true $M_{\gamma}=1$ event as $M_{c l}=2$ can be explained by the cross talking effect between crystals and clusters: $\gamma$-ray scatters from one crystal into another, which can be either adjacent or non-adjacent, in the latter case this effect increases $\mathrm{M}_{c l}$. The higher $\mathrm{M}_{\gamma}$, the stronger the cross talking effect which shifts $\mathrm{M}_{c l}$ to higher values.

On the contrary, the true $\mathrm{M}_{\gamma}=3$ event can be misidentified as $\mathrm{M}_{c l}=2$ event because the efficiency of detecting a single $\gamma$-ray is not $100 \%$. Thus, the higher $\mathrm{M}_{\gamma}$, the more $\mathrm{M}_{c l}$ is reduced.

These two processes, cross talking and loss due to efficiency, spread the $\mathrm{M}_{c l}$ distribution. For DANCE, loss due to efficiency affect $\mathrm{M}_{c l}$ more than cross talking, thus, the median $<\mathrm{M}_{c l}>$ tends to fall behind the true $\mathrm{M}_{\gamma}$ more as it increases: $\mathrm{M}_{\gamma}=3$ and $\mathrm{M}_{\gamma}=8$ correspond to $<\mathrm{M}_{c l}>=2.62$ and $<\mathrm{M}_{c l}>=6.2$ respectively; the ratios are $\frac{M_{\gamma}}{<M_{c l}>}(3)=1.145$ and $\frac{M_{\gamma}}{<M_{c l}>}(8)=$ 1.290 , these ratios do not change much as $\mathrm{E}_{\gamma}$ changes. 


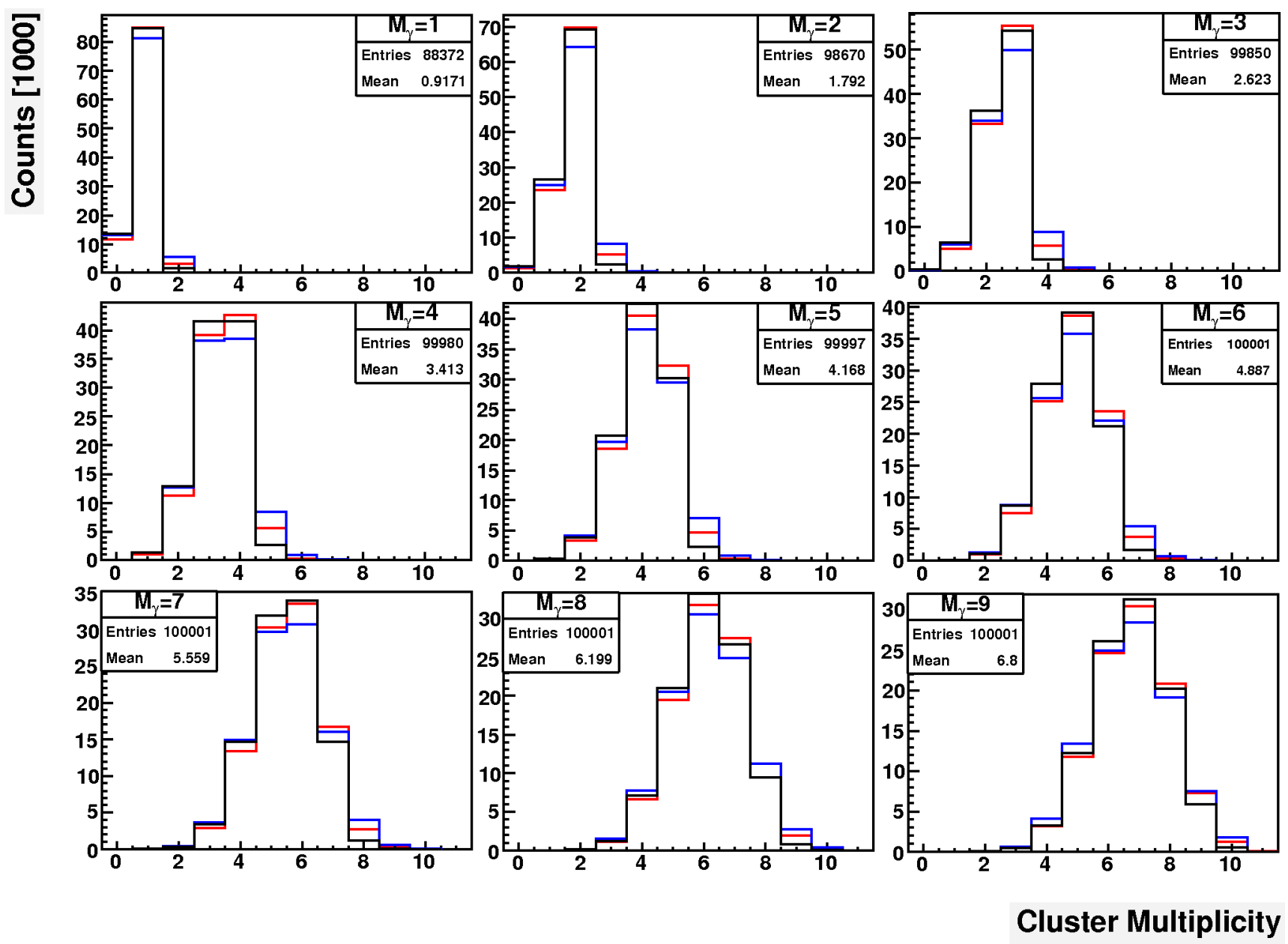

FIG. 24: The $\mathrm{M}_{c l}$ spectra for different $\mathrm{E}_{\gamma} .<\mathrm{M}>$ mean value is a function of $\mathrm{M}_{c l}$. The spectrum integrals (Entries) do not include the $\mathrm{M}_{c l}=0$ counts.

\section{DISCUSSION}

The nearly energy independent of the DANCE efficiency and multiplicity response to $\gamma$ rays is validated through the comparison between the measurement and numerical simulation for three radioactive sources. These capabilities of the DANCE array make it practical to compare the prompt fission $\gamma$-ray multiplicity distribution between the measurement and prediction after correcting for the detector response, thus establish the validity of the modeling the $\gamma$-ray production in fission. For the ${ }^{252} \mathrm{Cf}$ spontaneous fission, Brunson [? ] has established a mathematic model for the prompt $\gamma$-ray multiplicity distribution through his measurement. The figures Fig. 25 and 26 show how the initial $\mathrm{M}_{\gamma}^{f}$ distribution of Brunson model (black line) was folded with the DANCE response using the GEANT4 model and the resulting $\mathrm{M}_{c l}$ distributions are shown for three different energies: $\mathrm{E}_{\gamma}=0.511,1.0,2.0$ 
$\mathrm{MeV}$. The $\mathrm{M}_{c l}$ distributions are almost independent of $\mathrm{E}_{\gamma}$, which allows us to fold the $\gamma$-ray multiplicity distribution regardless the detailed $\gamma$-decay in fission.

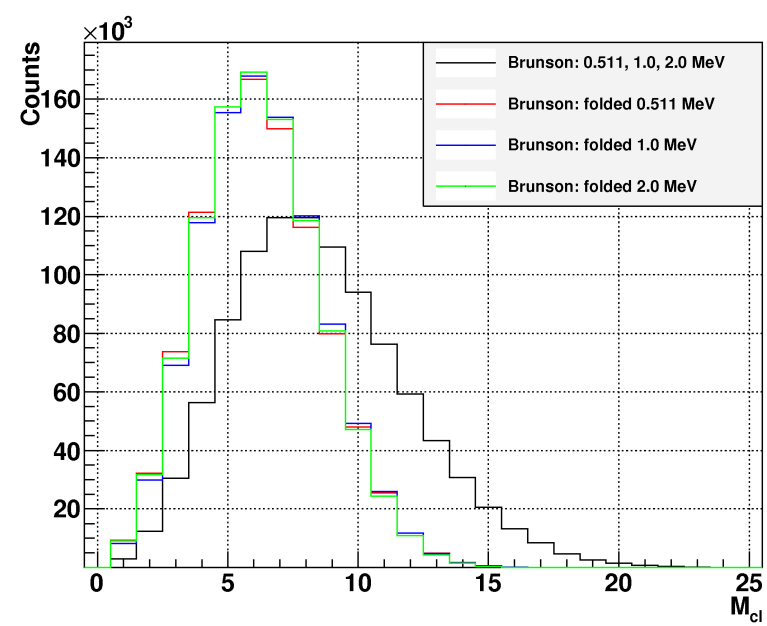

FIG. 25: Fission $\gamma$-ray multiplicity. Linear scale.

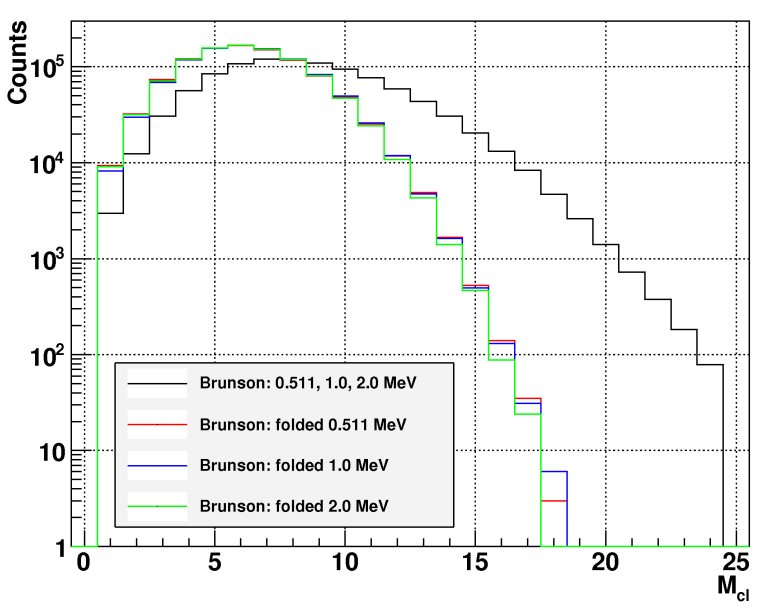

FIG. 26: Fission $\gamma$-ray multiplicity. Log10 scale.

We would like to take advantage of the DANCE array to establish a systematic understanding of the prompt $\gamma$-ray multiplicity distribution in fission. First, we plan to verify the Brunson model by measuring the prompt $\gamma$-ray multiplicity distribution for the ${ }^{252} \mathrm{Cf}$ spontaneous fission in the early 2011 when the beam is off. Second, we plan to measure the prompt $\gamma$-ray multiplicity distribution for the neutron-induced fission in ${ }^{239} \mathrm{Pu}$ and ${ }^{241} \mathrm{Pu}$. A newly designed fission counter under the NA22 funding will be used to differentiate the fission from the capture event. Both neutron-induced measurements have been scheduled during the period between September and October 2010. 